\title{
A New Method for the Application of Fiber to the Home in Internet Plus Era
}

\author{
DING Jing ${ }^{\star 1, a}$ \\ ${ }^{1}$ Xi'an fanyi university, Xi'an, China \\ Shuhua_y@126.com
}

Keywords: New Method; EPON; MPCP; LLID; QoS

\begin{abstract}
The new phase of Internet plus era promotes technical progresses of network transmission. Fiber to the home (FTTH) scheme is selected in many methods for solving the last mile of Internet transmission. At present, in view of the obstructions in applying FTTH, a FTTH application scheme is proposed based on Ethernet passive optical networks (EPONs). By using the multiple logical link identifiers (LLIDs), this technology allocates bandwidths according to service level agreements (SLAs) to meet the demands of different businesses for quality of service (QoS) and therefore well solves the problems of EPONs in wide application of FTTH. Through the actual operation of FTTH, the scheme is proved to be reliable.
\end{abstract}

\section{Introduction}

In the background of Internet plus era, it is critical to construct a networked, digitalized, personalized and lifelong education system, so as to build a learning society for everyone at anytime and anywhere and create a new environment for fiber to the home (FTTH). FTTH, as the most popular broadband access method, has not been widely used.

Ethernet passive optical networks (EPONs) have drawn more and more attention from network operation companies. However, due to the costs, operation maintenance, service quality, network management and insufficient demands of end users for bandwidths, EPON is commonly used as a supplement way by network operators for enterprise buildings and high-grade residential districts ${ }^{[1}$, 2].

Combining with EPON, currently a mainstream technology for fiber access, this study proposes a scheme for significantly promoting FTTH application. This EPON based scheme which is from multiple logical link identifiers (LLID) provides services with service level agreements (SLAs) for end users and guarantees the quality of service (QoS) for different businesses. Therefore, the scheme is able to meet the demands of current and future FTTH users.

\section{Major technologies of EPON}

Under the specifications of 802.3 media access control (MAC), new architectural standards are introduced. To begin with, the highly efficient data transmission and mature processing technology of Ethernet is transferred to easy to maintain passive optical networks (PONs) with low costs. Furthermore, dual wavelength structures $(1,550 \mathrm{~nm}$ and 1,310 $\mathrm{nm}$ for downstream and upstream, respectively) and time-division multiplexing (TDM) technology are used to share bandwidths and broadcast transmission is encrypted for different users. In this way, the rate of upstream and downstream lines can reach $1.25 \mathrm{Gbit} / \mathrm{s}$. It is critical for EPON technology to solve the control of multiple optical network units (ONUs) in PONs on the upstream transmission of a same optical line terminal (OLT). Therefore, the Ethernet in the first mile (EFM) defines the multi-point control protocol (MPCP) in IEEE802.3ah standard and expands the MAC control sub-layers in IEEE802.3. MPCP sets a control mechanism to coordinate the efficient delivery and receipt of data between master and slave devices with point to multipoint (P2MP) and uses messages, state machines and timers to control topological structures for accessing P2MP. The automatic discovery and registration 
process of ONUs and dynamic bandwidth allocation (DBA), query and monitoring are defined. MPCP is a support protocol and not related to high-level contents including bandwidth allocation strategy, access authentication, service provision and management, and the definition of QoS ${ }^{[3,4]}$.

MPCP is located in MAC control sub-layers. The layered system in the side of OLTs virtualizes multi-MAC entities which share a same physical layer. Moreover, each MAC entity provides a P2P simulation service between OLTs and ONUs. In addition, OLTs virtualize a special single copy broadcast (SCB) of MAC entity and simultaneously communicates with all ONUs by sending a SCB frame received by all ONUs. In the side of ONUs, each ONU only needs to virtualize a MAC entity, so as to communicate with a MAC entity in OLTs. In this way, a P2P simulation layer of EPONs is formed on P2MP platform. By adding a double-byte LLID in the leading code of each datagram, the topologies of P2MP network are simulated as a set with multiple P2P links.

The frame length of EPONs is changeable and the frame structures are similar with Ethernet frames. The difference is that 5 new control frames with 64 bytes are introduced. They are GATE and REPORT frames for allocating and requesting bandwidths and REGISTER frames including REGISTER, REGISTER_REQ and REGISTER_ACK for controlling automatic discovery process. In order to maximize compatibility with Ethernet, they adopt the basic 88-08 type Ethernet frames without labels. These control frames are inserted by MPCP functional entities.

\section{MAC protocol of EPONs based on FTTH}

At present, in the applied MPCP mechanisms, ONUs only need to virtualize a MAC entity, that is, ONUs correspond to LLIDs, and the system structures are shown in Figure 1. Aiming to adapt to the current development of FTTH, a multiple LLID scheme is proposed. In other words, ONUs can virtualize multiple MAC entities or an ONU can allocate multiple LLIDs ${ }^{[5,6]}$. The number of LLIDs is determined by that of Ethernet ports contained in ONU devices (LLIDs correspond to Ethernet ports of ONUs) and each LLID corresponds to a user. In this way, OLT can directly manage single users according to LLIDs and serve single users in accordance with SLAs. Furthermore, in order to satisfy QoS demands of users and ensure the service quality of different businesses, each LLID is divided into three service levels including expedited forwarding (EF), assured forwarding (AF) and best effort (BF) services. These three service levels correspond to three business types including voice, video and data. The system structure is demonstrated in Figure 2 and the technical characteristics of this scheme are illustrated in the following in detail ${ }^{[5,6]}$.
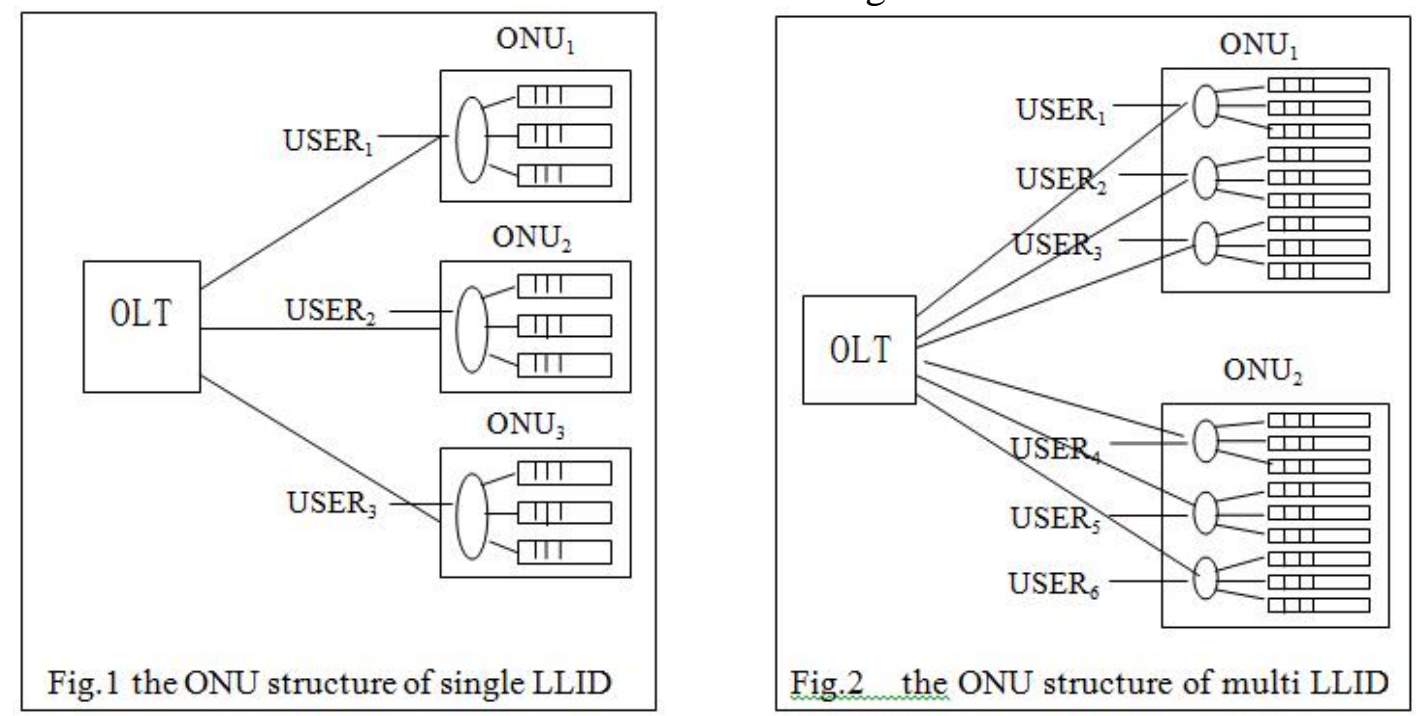

Registration. Traditional ONUs are registered after starting OLTs and an active ONUs is found in the automatic operation discovery process. Therefore, OLTs allocate a LLID to the ONU and the discovery process is displayed in Figure 3. 
This study carries out automatic discovery of ONUs and LLID allocation in two steps. Firstly, the automatic discovery of ONUs is conducted. When ONUs are active, LLIDs are not allocated immediately but it is necessary to check whether there are active Ethernet ports on ONUs. If an Ethernet port is detected to be active, ONUs need to virtualize a MAC entity to communicate with MAC entities in the side of OLTs. Secondly, OLT allocates a LLID to MAC in ONUs. Moreover, ONUs pre-allocate three storage spaces under this LLID to store three types of business data in different priorities. Owing to the data traffics of the three types of businesses are different, the storage spaces is allocated based on business types: $128 \mathrm{~K}$ for voice business, 2-8 $\mathrm{M}$ for video business and 0-10 $\mathrm{M}$ for data business. Therefore, the number of active Ethernet ports of ONUs determines the number of LLIDs. The discovery process of each MAC is the same with the register process of a single LLID.

Dynamic bandwidth allocation scheme. The proposed dynamic bandwidth allocation scheme still adopts traditional GATE/REPORT mechanism. Assume that $\mathrm{n}$ users apply for LLIDs and each user is labeled as LLID . Because this scheme mainly aims at FTTH, SLAs of each user are guaranteed. According to SLAs, users are classified into different levels and the rotation table is made to directly allocate bandwidths to single users. The rotation table consists of LLID labels of active users, Buy Band of users, Mini Band, EF bytes, AF bytes, BF bytes and round-trip time (RTT), as shown in Table.

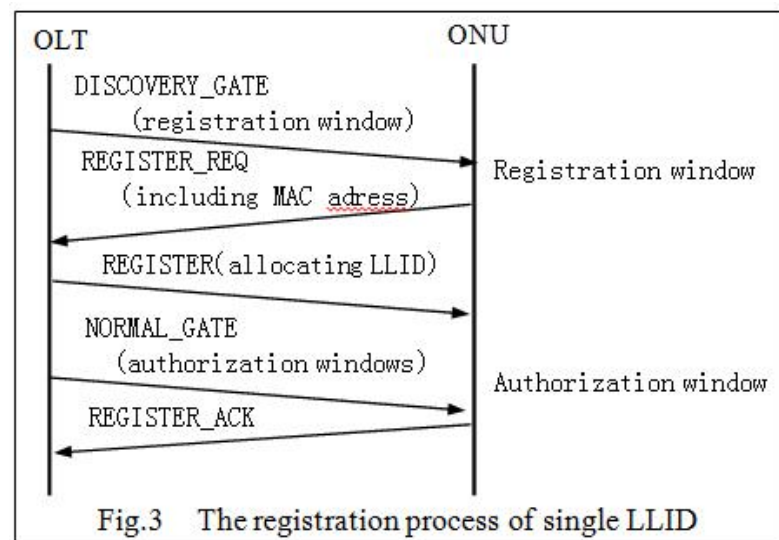

\begin{tabular}{|c|c|c|c|c|c|c|}
\multicolumn{7}{|c}{ Table1. OLT polling } \\
\hline 1 & $B b_{1}$ & $M b_{1}$ & $B e f_{1}$ & $B a f_{1}$ & $B b f_{1}$ & $T_{1}$ \\
\hline 2 & $B b_{2}$ & $M b_{2}$ & $B e f_{2}$ & $B a f_{2}$ & $B b f_{2}$ & $T_{2}$ \\
\hline$\ldots$ & $\ldots$ & $\ldots$ & $\ldots$ & $\ldots$ & $\ldots$ & $\ldots$ \\
\hline $\mathrm{i}$ & $B b_{\mathrm{i}}$ & $M b_{\mathrm{i}}$ & $B e f_{\mathrm{i}}$ & $B a f_{\mathrm{i}}$ & $B b f_{\mathrm{i}}$ & $T_{\mathrm{i}}$ \\
\hline$\ldots$ & $\ldots$ & $\ldots$ & $\ldots$ & $\ldots$ & $\ldots$ & $\ldots$ \\
\hline $\mathrm{n}$ & $B b_{\mathrm{n}}$ & $M b_{\mathrm{r}}$ & $B e f_{\mathrm{n}}$ & $B a f_{\mathrm{n}}$ & $B b f_{\mathrm{n}}$ & $T_{\mathrm{n}}$ \\
\hline
\end{tabular}

The specific processes of dynamic bandwidth allocation based on GATE/REPORT mechanism are not described in detail but shown in Figure 4. The following points need to be noticed.

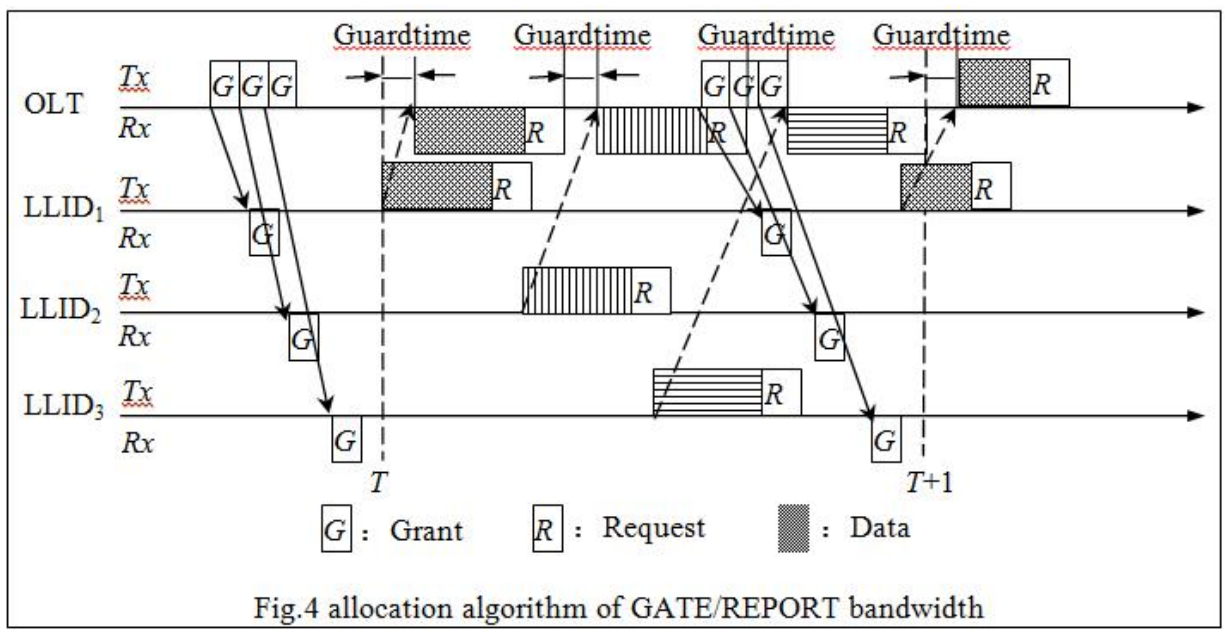

Bandwidth allocated to users is not more than the Buy Band in principle. However, when there is residual after allocating bandwidths (according to Buy Band of users), the allocated bandwidth to users (the demands are more than Buy Band) can exceed Buy Band. The excess bandwidths are allocated to users in need in accordance with the proportion of Buy Band. 
The Buy Band of users and the Mini band satisfy the regulations of SLAs. Mini Band= Buy Band $\times \alpha(\alpha<1$, operators' setting).

Rotation cycle is $2 \mathrm{~ms}$ and $1 \mu$ s is used for guard band.

Request frame REPORT includes request bandwidths for three businesses of a user, while authorization frame GATE consists of authorization bandwidths for three businesses and the start time for sending. The allocation principle of bandwidth is to allocate bandwidth according to service levels. Firstly, EF service is allocated in accordance with needs and then the bandwidth of AF service is assigned. Finally, the residual bandwidths are allocated to BF service. The allocation proportion is set based on Buy Band.

\section{Conclusions}

In view of some problems of EPONs currently appearing in the development of FTTH, this study proposes a transitional scheme with several advantages. Owing to an ONU is shared by multiple users, the cost is shared and therefore the costs for single users decrease. Each user utilizes a LLID and OLTs directly manage single users. Moreover, according to SLAs, different bandwidths are allocated to users, which meet the demands of different users for bandwidths and are easy to charge. Each user has three different businesses, satisfying users' QoS demands of different business types. As users increase bandwidth requirements, this scheme can transit smoothly to suit for future development of FTTH. However, there are also disadvantages. Because one ONU supports multiple users, the design complexity and costs increase. Furthermore, the occupied bandwidths by authorization frame and guard band under the structure of multi-LLID increase, which reduces the bandwidth utilization.

Although this scheme is at the cost of increasing the complexity of ONUs, it can well solve the problems existing in FTTH development. So, it is applicable to the current FTTH development, meets the bandwidth needs of different users, and ensures the service quality of different businesses. With the constantly increasing demands of end users for bandwidths, the scheme can transit smoothly through slight transformation of equipments, so as to fit FTTH development demands and save a large number of funds. Therefore, this is a practical and ideal scheme.

\section{Acknowledgement}

In this paper, the research was sponsored by the nature science research projects of education department in Shaanxi Province government and key project of education department in Shaanxi provincial government (Project No. 13BZ69).

\section{References}

[1] LIU Shan Hua. Application and research in telecom access network Based on FTTH [J]. Telecom World,2016(09):75-76.

[2] QU Huai Yu. Discussion on Taiyuan FTTH Network Building Solution [J]. Video Engineering,2015(06):43-47.

[3] IEEE 802.3ah Ethernet in First Mile Task Force Presentation Materials

http://grouper.ieee.org/groups/802/3/efm/public/.

[4] ZHANG Jian Ping,CHEN Yi,ZHANG Jie Xin. The research of key technologies and network structures based on channel service in FTTH network [J].Telecommunications science, 2012(11):126-131.

[5] G.Kramer,B.Mukherjee, and G pesavento, "Interleaved Polling with Adaptive Cycle Time (IPACT):A Dynamic Bandwidth Distribution Scheme in an Optical Access Network,"Photonic Network Comm, 2002,4(1):89-107. 
[6] XIAO Wen Long. Design and implementation in telecom access network Based on GPON technology [J]. Telecom World, 2016(16):32-33. 